\title{
Enhancing Multi-Objective Optimization for Wireless Sensor Networks Coverage using Swarm Bat Algorithm
}

\author{
Ibrahim A. Saleh \\ Dept. of Software Engineering \\ College of Computer Sciences \& Math \\ University of Mosul, Iraq
}

\begin{abstract}
Coverage area in wireless sensor network (WSN) is very important for network's performance.it has several critical and challenges that are to be occupied when designing the techniques and algorithms to increase the Network lifetime. Therefore WSN poses problem involve exchange data between multiple conflicting optimization objectives such as coverage preservation. The proposed paper applies new approach to optimize the coverage performance of WSN. The algorithm strategy new multi-objective optimization bat swarm algorithm with adaptive neighborhood processes and turnoff redundant sensor nodes. Any position of mobile sensor nodes represented by bat which is used in hybrid bat algorithm. The algorithm is presented an adaptive neighborhood which can successfully avoid possibility the turnoff redundant sensor. Simulation results show that experimental results can able to improve coverage of WSN, increase the time life of network and low energy consumption
\end{abstract}

\section{Keywords}

Wireless Sensor Network, Bat algorithm, Multi -objective Optimization, Coverage, Neighborhood disturbance, redundant node

\section{INTRODUCTION}

Wireless sensor networks (WSN) consist of a lot of numbers of sensor and mobile nodes that have multi-hope wireless network manner, limited processing, small size, tiny power and short distance communicate. All WSNs are design to cover maximizes area of a targeted region with high lifetime of network. The WSN features are deployed with high density, random deployment, ability to handle with node failures; mobility of nodes, scalability and ease of use [1] .Each sensor node has limited storage capacity, computation power, and bandwidth and power supply. Deference sensors embedded in the node measure environment parameters, collect data through nodes and transmit to his network or through the upper network [2]. Energy efficiency is directly related with efficient routing of data. The clustered of WSNs are naturally used to reduce the communication overhead, thereby decreasing the energy consumption and interference among the sensor nodes [3].

The sensor nodes are distribution randomly to perform specific key objectives such as: wireless covered area, area monitoring, biological detection, weather reporting ...etc. Sensor nodes are connected together to create network for covering specific area [4].Therefore, it happen coverage overlap between some nodes in most areas where same work that lead result will in waste of energy and shorten network's life . On the other hand, one of the key issues in some area of wireless sensor networks is monitoring the vacuum network coverage [5], while WSN is deployed randomly and it needs improvement coverage area [6]. For that, there is a problem worthy to study the following:

Random deployment of sensors will lead to overlapping coverage areas. Therefore, changing position of mobile sensor should also be found as far as possible overlapping areas.

The redundant sensor nodes in the region into a sleep state also they need covered by other sensor nodes to improve high life of the network [7].

One of the solving above problems is applied multi-objective optimization (MOO) also called (Pareto optimization). The concept of multi- objective numbers of standards choice are search for many targets or if there is more than one targets need to process at same time and improved between the various objectives such as limited numbers of sensors are covered large region of area in network [8]. The problem concerned is involving more than one objective function to be optimized simultaneously, generally the basic MOO equation is formulated as follow [9]:

$$
\min _{X \in \Omega} F(X)=\left(F_{1}(X), F_{2}(X) \ldots . F_{L}(X) \ldots \ldots(1)\right.
$$

Where is: $\boldsymbol{X}$ is a possible solution, $\boldsymbol{\Omega}$ is feasible solution space, $\boldsymbol{F}($.$) is the objective function and \boldsymbol{F}_{\boldsymbol{r}}($.$) is r^{\text {th }}$ objective function for $\mathbf{1} \leq \boldsymbol{r} \leq \boldsymbol{L}$ ). A solution dominates solution $\boldsymbol{b}$ (or $\boldsymbol{b}$ is dominated by $a$ ) if satisfied the following condition:

$$
\boldsymbol{F}_{i}(\boldsymbol{a}) \leq \boldsymbol{F}_{\boldsymbol{i}}(\boldsymbol{b}), \quad \forall \boldsymbol{i} \in\{1,2, \ldots \ldots \ldots L\}
$$

A solution is called non-dominated solution, if it is not dominated by any other one solution" when $\boldsymbol{a}$ is uninterested to solution $\boldsymbol{b}$, if $\boldsymbol{a}$ cannot dominate $\boldsymbol{b}$, and $\boldsymbol{b}$ cannot dominate $\boldsymbol{a}$. in this state Pareto-optimal set is constituted of nondominated solutions [10]. Therefore, the problem has multiple optimal solutions. it must need decision be chosen best one among them, depending on criteria priorities of the objectives to be achieved and degree of preference for multiple objectives [11 For monitoring area by WSN, sensor's nodes distributed randomly in the area. Sink node (base station) received monitor data from all nodes through multiple hops and forward aggregated data packets. In this state the applications acting two methods first, the communication link between sink nodes which collect the data, second the sensor's nodes also communication among them and work together [12].

The term "coverage" refers evaluate metric for wireless network ability to deploy a network over a larger physical area to increase a system's value to end user. Multi-hop communication techniques can extend coverage of network well beyond to range of the radio technology alone. The aim of coverage problem is to use minimum number of sensors which cover the entire target region [12]. Fig (1) illustrates a coverage area of WSN node. 


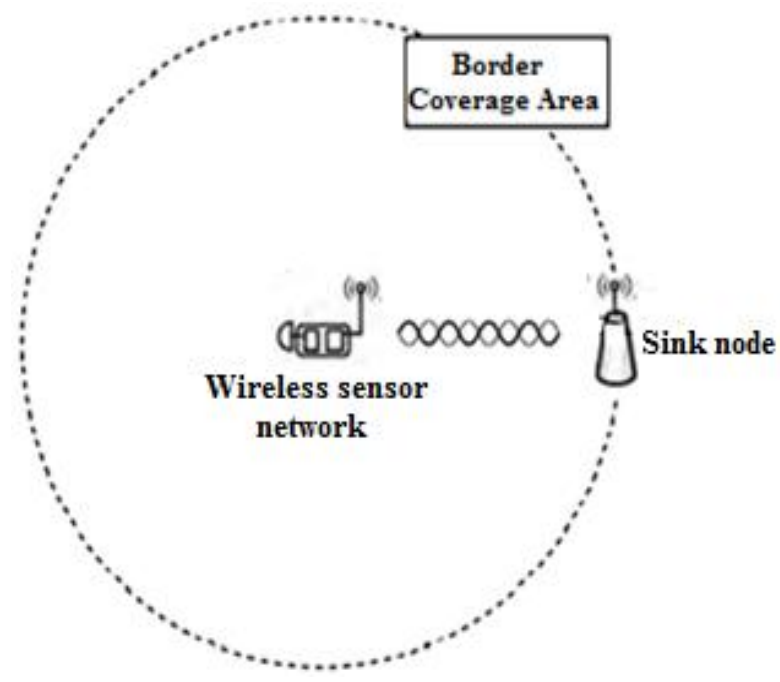

Fig (1): Coverage area for WSN

In old WSN topology, nodes distributed randomly in monitored area. Nodes together are contained selforganization formula in the network. All nodes send the monitor data to sink node through multiple hops, finally transmit data to the remote center. In new topology of WSN uses, acting temporary link between sink nodes communication, nodes worked together and gathered data and also exists data communication among nodes [13].

This paper is proposed a multi-objective evolutionary algorithm based on new optimization method bat algorithm (BA) [14] and apply it on optimizing of several objectives simultaneously. BA is used for fixed sensors and mobile nodes to build a combination of hybrid wireless transmission sense network. These techniques are used in many applications such as monitoring accurately for dangerous environment (like toxic chemical zone, radiation zone, etc.) [4].

The paper is studied how to maximize scope of monitoring wireless sensor networks and improve network coverage network deployment strategies. The basic policy of nondominated sorting is improved by multi-objective based on BA. The optimization intelligence algorithm is particle sub analog of mobile node position. It combined with redundant nodes detected on results of each particle iteration take adaptive neighborhood scrambling to adapt based on move of weighted value and then select optimum particle participation from next evolution, so as to improve the coverage of wireless sensor networks and extend network lifetime goal. The rest of this paper is structured as follows: In section 2 described the proposed methodology which includes network modeling and redundant nodes detecting. Section 3 describes briefly bat swarm algorithm of multi optimization solution. In section 4, some simulation results are presented and finally, in section 5 conclude the paper.

\section{PROPOSED METHODELOGY}

\subsection{Network Model}

To design model with multi-objective optimization for WSN. At first, consider $\boldsymbol{A}$ is a discrete target rectangular monitoring area randomly .It plans into grid a two-dimensional $\boldsymbol{L} \times \boldsymbol{W}$. Each grid represents a small area to be monitored in area $\boldsymbol{A}$. put $\boldsymbol{M}$ mobile sensor nodes and fixed hybrid wireless sensor networks $\boldsymbol{N}$ sensor nodes randomly. which looks similar the one shown in fig(2). Under mention, the algorithm does the following assumptions:
1. Each node sent data to other nodes via an appropriate route to sink, consider size of packets is identical.

2. The energy of sensor nodes is fixed and when its energy is depleted the node does not work.

3. All sensor nodes have the same function and equal status in any way except one node called "sink node" which has unlimited energy.

4. The sections of all wireless communication range is based on center's node point as condition to circular area with a radius of $\mathrm{Rc}$, the node perception radius $\mathrm{Rs}$ and $\mathrm{Rc}=2 \mathrm{Rs}$;

5. Each node communication model uses Boolean communication model.

6. Each node has three kinds of jobs (active, detection and dormant state).

7. Coverage of redundant node area can be completely by other neighbor sensor nodes.

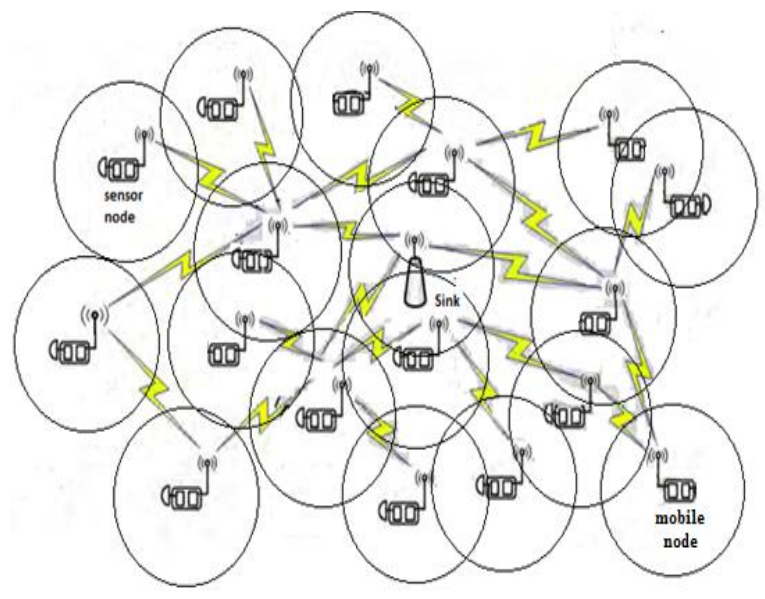

Fig (2): Distributed of sensor node network

The distance $\boldsymbol{d}$ of nearest neighbor sensor node $\boldsymbol{s}_{\boldsymbol{i}}\left(\boldsymbol{x}_{\boldsymbol{i}}, \boldsymbol{y}_{i}\right)$ in $\boldsymbol{K}$ calculate either within the target area $\boldsymbol{A}$ or its center coordinates $\left(\boldsymbol{x}_{\boldsymbol{k}}, \boldsymbol{y}_{\boldsymbol{k}}\right)$ is defined as:.

$$
d\left(k, s_{i}\right)=\sqrt{\left(x_{k}-x_{i}\right)^{2}+\left(y_{k}-y_{i}\right)^{2}}<R_{i} \ldots .
$$

the probability of cover point sensor $\boldsymbol{k}_{\boldsymbol{s}}$ is perceived $\boldsymbol{P}(\boldsymbol{k}, \boldsymbol{s})$ = 1; Otherwise, $\boldsymbol{P}(\boldsymbol{k}, \boldsymbol{s})=\mathbf{0}[6]$, the $\operatorname{grid} \boldsymbol{I}\left(\boldsymbol{x}_{\boldsymbol{k}}, \boldsymbol{y}_{k}\right)$ in grid set set $X=\left\{s_{1}, \ldots, s_{n}\right\}$ defined as :

$$
I(k, X)=1-\prod_{i=1}^{n}\left[1-P\left(k, s_{i}\right)\right]
$$

Accordingly, the total coverage of wireless sensor networks is doing by the grid points set $\boldsymbol{X}$, denoted $\operatorname{Cov}(\boldsymbol{X})$, the network is operating sensor nodes covered is given by:

$$
\begin{gathered}
\operatorname{Cov}(X)=I(k, X) \ldots \\
P_{\operatorname{Cov}(X)}=\frac{\operatorname{Cov}(X)}{L * W} .
\end{gathered}
$$

$\boldsymbol{P} \_\boldsymbol{C o v}(\boldsymbol{X})$ for the hybrid wireless sensor networks coverage of the target area $\boldsymbol{A}$. The number of sensors dormant written as:

$$
\operatorname{Sleep}(X)=N+M-|X|
$$

Where $|\mathrm{X}| \mathrm{X}$ represents the number of elements in set. 
If the operating state of sensor nodes as possible as little and area covered by target area as possible as large [15]. Thus, hybrid radio sensor network coverage problem can be attributed to satisfy fully meshed network conditions i.e. operating condition for any sensor node $\boldsymbol{s}_{\boldsymbol{i}}\left(\boldsymbol{x}_{\boldsymbol{i}} \boldsymbol{y}_{i}\right)$, presence. A sensor $s_{j}\left(x_{j}, y_{j}\right)$ is working to cover the area of two objective optimization, such that

$d\left(s_{i}, s_{j}\right)=\sqrt{\left(x_{i}-x_{j}\right)^{2}+\left(y_{i}-y_{j}\right)^{2}}<R_{c}$

To define establishment of two-objective optimization illustrate in equation (7)

$$
\begin{gathered}
\max F(X)=\left[f_{1}(X), f_{2}(X)\right] \text { s.t. } \forall i \epsilon[1, N+M], \quad \exists j \in \\
{[1, N+M], \text { and } i \neq j \ldots(7)} \\
\text { Where: } f_{1}(X)=\operatorname{Cov}(X), f_{2}(X)=\operatorname{Sleep}(X)
\end{gathered}
$$

\subsection{Redundant nodes detecting}

Although the sensor network nodes are random deployment. It is very easy to awareness overlapping from a coverage area. Any sensor's node can be shut down for any time for network's service life. In this paper, practical detection method to schedule turns off or start sensor from nodes adaptive sleep [16]. There is specific detection methods are working for following:

1. First, there is a random set of sensor's nodes in a dormant state and other nodes in work status.

2. Each node after a period of dormancy has random sleep time $\boldsymbol{t}_{\boldsymbol{s}}$. It wake up and detect their diameter within $\boldsymbol{R} \boldsymbol{s}=$ 0.8 , whether if there are other work nodes in area determine this node work or not.

3. After waking up, the appropriate node must working power transmission and send "PROBE" message to sink nodes.

4. The sink node in this region is spread "REPLY" to all neighborhood nodes in network after receives the above message.

5. If dormant node not listens to REPLY messages. It adjusts the time of sleep room and sleeps.

6. If nodes receive REPLY message within a period of time. It transferred it from sleep state to work state. fig (3) illustrate the states of nodes

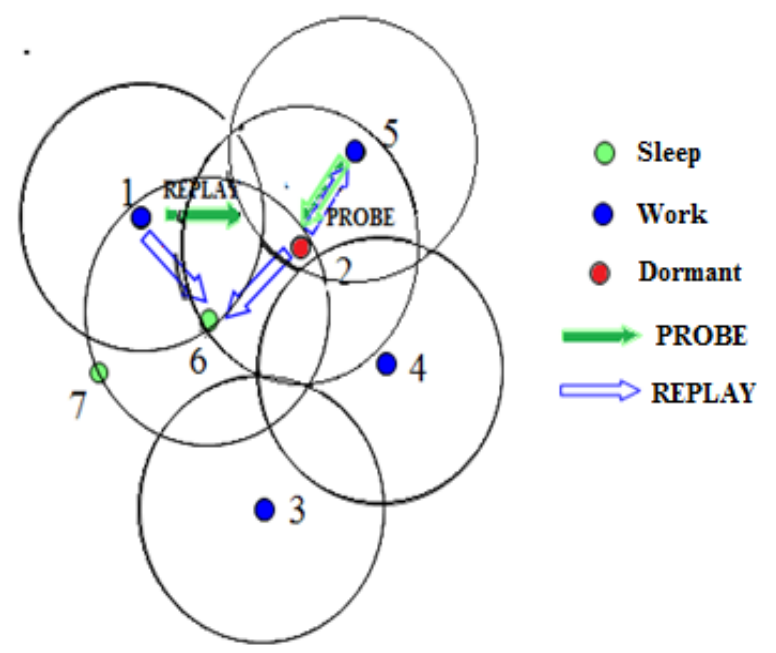

Fig (3): Nodes States

\section{MULTI-OBJECTIVE COVERAGE OPTIMIZATION ALGORITHM \\ 3.1 Bat algorithm}

Bat algorithm (BA) and its various improved algorithms [17] have been successfully applied in many fields optimization problem and get a single goal in the field of great success. BA balances the global search and local search by adjusting loudness and pulse rate. It is based on behaved of natural bats. Bats are animals that have wings and possess the capability of echolocation. Echo locating animals emit calls to the environment and listen to the echoes of those calls. These echoes will be used to locate and identify objects. With this echo a bat can determine an object's size, shape, direction, distance, and motion. When the bats fly close to their prey a constant frequency in each pulse is also observed. The loudness for searching for prey is greater than when homing towards the prey. In other words, the loudness decreases when getting nearer to the victims. BA simulated parts of the echolocation individualities of micro-bat in simplicity way as the follows.

- $\quad$ All bats utilized echolocation to detect the prey.

- There are special types of bats called "micro-bat" which is widely using echolocation.

- A swarm of bats fly randomly with velocity $\boldsymbol{V}_{\boldsymbol{i}}$ at position $\boldsymbol{X}_{i}$ with a fixed frequency $f$, varying wavelength $\lambda$ and loudness $\boldsymbol{A}$ to search for a prey.

- Bats have ability to adjust the wavelength of their emitted pulses and regulate the rate of pulse emission $\boldsymbol{r} \in[\mathbf{0}, \mathbf{1}]$ depend on the immediacy of their target.

- The loudness can be changed from a large (positive) $\boldsymbol{A}_{0}$ to a minimum constant value $\boldsymbol{A}_{\min }$. Also frequency $f$ is in range $\left[f_{\min }, f_{\max }\right]$ matches to a range of wavelengths $\left[\lambda_{\text {min }}, \lambda_{\text {max }}\right]$.Fig (4) shows the basic algorithm for BA [18]. 
Object function $f(x)=\left[x_{1}, x_{2}, \ldots \ldots x_{d}\right]^{T}$

Step 1: Initialize the bat population location $\left(x_{i}\right)$ and velocities $\left\{V_{i}(i=1,2, \ldots \ldots, n)\right\}, \beta$ is a random vector is drawn from a uniform distribution

Find the current best bat $\left(x_{*}\right)$

Step 2: Define pulse frequency $\left(f_{\max }, f_{\min }\right)$ Initialize pulse rates $\left(r_{i}\right)$ and the loudness

$\left(A_{i}\right)$

Step 3: While $(t<$ Max number of iterations)

Generate new solutions by adjusting

frequency,

locations/solutions

$$
\text { and updating velocities and }
$$

$$
\begin{gathered}
f_{i}=f_{\text {min }}+\left(f_{\text {max }}-f_{\text {min }}\right) \beta \\
v_{i}^{t}=v_{i}^{t-1}+\left(x_{i}^{t}-x_{*}\right) f_{i} \\
x_{i}^{t}=x_{i}^{t-1}+v_{i}^{t}
\end{gathered}
$$

Step 4: If $\left(\operatorname{rand}(0,1)>r_{i}\right)$

Select a solution among the best solutions

Generate a local solution around the

selected best solution

End if

Generate a new solution by flying randomly

If (rand $\left.<A_{i} \&\left(x_{i}\right)<(x)\right)$

Accept the new solutions

Increase $r_{i}$ and reduce $A_{i}$

End if

Rank the bats and find the current best $x_{*}$ End while

Step 5: Post process results and visualization.

\section{Fig (4): Basic Bat Algorithm}

In this paper apply hybrid bat algorithm which used multi-bat based on many swarms are implemented simultaneously that lead to the parameters (Frequency, velocity and location) are parallel updated. The many swarms have different control parameter settings to give all one of them his search result. The multi-swarm exchange information among them through immigration operator and gathered results for the final optimal solution. The multi- swarm co-evolution select the finest individual in each population through selection operator on each generation [19].

\subsection{Multi-objective a Neighborhood Disturbance with BA}

Bat algorithm has been proven a good optimal solution with a smaller computational cost for solution neighborhood disturbance nodes. However, it is optimized for multi-target [20] still need to address the following issues:

1. How to generate non-dominated solutions in the search process using constitutes a Pareto solution set.
2. What kind of policy from the current non-dominated solutions?

3. Select the global optimum focus (or local optimization) particles.

4. How to maintain diversity on the Pareto front optimization solution?

The hybrid bat algorithm for multi-objective a neighborhood disturbance is used to solve the above assumptions. At first conduct the network optimization to generate initial individual to adopt the optional multi-objective to generate the initial population and do the following:

- Multi-band neighborhood is based on the front-end f1 from non-inferior to non-dominated sorting strategy in disturbance objective bat algorithm.

- $\quad$ Search operator with different parameter settings is trade-off information between swarms that lead the algorithm can do a best exchange between global and local search.

- Weighting method is used from different swarms to choose best global particle randomly.

- Each bat has their own local optimum particles from particle in motion based on role update in position and their fitness adaptive neighborhood disturbances.

\section{COVERING OPTIMIZATION HYBRID WIRELESS SENSOR NETWORK}

\subsection{Encoding method}

This hybrid wireless sensor networks has $\boldsymbol{N}$ from random fixed sensor nodes and $\boldsymbol{M}$ mobile nodes are delivered information from target region. The covering optimization in network can be as much as possible to adjust position of mobile nodes and can monitor is covered a larger area [21]. There are also dormant nodes as possible can have more deployment. Mobile scenario $\boldsymbol{M}$ nodes with a particle group particles representatives $Z_{k}^{i}=\left(Z_{1}^{i}, Z_{2}^{i}, \ldots \ldots \ldots, Z_{M}^{1}\right)$ on behalf of the $i^{\text {th }}$ microbat, where $X_{i}=\left(X_{k}^{i}, y_{k}^{i}\right)$ means former position for first mobile node $\boldsymbol{k}$.

\subsection{Selection of the best individuals}

To select the best individuals by applied BA in WSN. The fitness function $\boldsymbol{F i t}(\boldsymbol{i})$ value of each individual node $\boldsymbol{i}^{\text {th }}$ in collection of different nodes is illustrated in equation (8). The weighed of the front-end $\boldsymbol{f} \boldsymbol{l}$ in individual non-inferiority with the largest individual fitness as a global optimal.

$$
\operatorname{Fit}(i)=\alpha * \operatorname{Cov}\left(X_{i}\right)+\beta * \operatorname{Sleep}\left(X_{i}\right)
$$

Where $\boldsymbol{\alpha}$ and $\boldsymbol{\beta}$ are adjustment coefficients, their values chose from network's designers depending to comprehensive requirements on indicators network performance,

$$
\alpha \in[0,1], \beta=1-\alpha \text {. }
$$

To updating the velocity and position of the $\boldsymbol{i}^{- \text {th }}$ particle, respectively, by the equation (9), (10) is completed.

$$
\begin{gathered}
v_{i}^{t}=v_{i}^{t-1}+\left(x_{i}^{t}-x_{*}\right) f_{i} \\
x_{i}^{t}=x_{i}^{t-1}+v_{i}^{t} \ldots \ldots \ldots \ldots
\end{gathered}
$$

Where: $X_{i}(\mathrm{t})$ and $V_{i}(t)$ denote the $\boldsymbol{i}^{\text {th }}$ particle position and velocity at time $t$. 


\subsection{Adaptive neighborhood disturbances}

Neighborhood disturbances scheme is shown in Fig (3). It is defined the disturbance of particle neighborhood for vector particles. It represents individual components within a preset range of random variable $\boldsymbol{\delta}$ neighborhood. To enhance the current position of the particle local search for the $\boldsymbol{m}^{\text {th }}$ component of the particle $\boldsymbol{Z}_{\boldsymbol{m}}$, its adaptive disturbance operation is defined as:

$$
Z_{m}^{i^{\prime}}=\left\{\begin{array}{c}
\left(\begin{array}{c}
x_{m}^{i}+\delta * e^{-F i t(i)} * L_{i}(k) *(-1)^{k} \\
y_{m}^{i}+\delta * e^{-f i t(i)} * L_{i}(k) *(-1)^{k}
\end{array}\right) \\
r_{i m} \geq \frac{F i t(t)}{f i t(\text { best })} \\
\text { else } \quad Z_{m}^{i}
\end{array}\right.
$$

\begin{tabular}{||c||c||c||c||}
\hline 75 & 70 & 90 & 6 \\
\hline \hline 100 & 72 & 94 & 5 \\
\hline \hline 125 & 62 & 75 & 8 \\
\hline \hline 150 & 58 & 70 & 9 \\
\hline \hline 175 & 68 & 82 & 7 \\
\hline \hline 200 & 70 & 95 & 6 \\
\hline \hline 225 & 78 & 98 & 5 \\
\hline \hline 250 & 75 & 94 & 5 \\
\hline
\end{tabular}

Where: $\boldsymbol{\delta}$ is the disturbance amplitude parameters,

$\boldsymbol{L i}(\boldsymbol{k})$ : the k Logistic sequence value,

$\boldsymbol{T}$ : maximum number of iterations,

$t$ : current iteration number,

$\boldsymbol{r}_{\text {im }}$ : random number which a value between $[0,1]$ is used to control whether the first particle $\boldsymbol{i}$ components $\boldsymbol{m}$ required disturbance can be seen from the equation (11)

The number of iterations is controlled by adaptation value and amplitude size. In initial iteration ( $\mathbf{T}$ is smaller) the disturbance is large. Meanwhile, if the individual fitness value is greater, then opportunities and amplitude perturbations.

\section{SIMULATION RESULT}

The proposed algorithm is simulated by Opnet- 14 to perform the scenario assumed with 40 nodes in square region of size $(100 \times 100)$. In which active node to be covered is a meter, with the 40 perception radius $\boldsymbol{R} \boldsymbol{s}=10$ using the following

1. Sensor nodes and mobile nodes deployed randomly hybrid wireless sensor networks, each node $=10 \mathrm{~m}$, communication radius $\boldsymbol{R} \boldsymbol{c}=20 \mathrm{~m}$.

2. Population size of bat algorithm is set to 20 , the maximum perturbation amplitude $\delta$ is set to $(0.8 \times \boldsymbol{R} \boldsymbol{s})$ namely $8 \mathrm{~m}$, parameters, loudness(A), pulse rate equal 0.5 , and frequency $f_{\min }=0, f_{\max }=2$.

3. Maximum number of iterations $\mathrm{T}=250$. Fitness function equation (6), Parameter Settings $\alpha=0.6, \beta=0.2$.

\subsection{Verify the Effectiveness and stability of the algorithm}

To verify the stability of the proposed algorithm, it compared between two scenarios: one with optimization using hybrid bat algorithm and another without. The dormant nodes are different for each iteration. The two algorithms run 10 times illustrated coverage rate in table (1)

Table (1): The coverage rate before and after optimization

\begin{tabular}{|c||c||c||c||}
\hline Iteration & $\begin{array}{c}\text { Coverage rate } \\
\text { before } \\
\text { optimization } \\
(\%)\end{array}$ & $\begin{array}{c}\text { Coverage } \\
\text { rate before } \\
\text { optimization } \\
(\%)\end{array}$ & $\begin{array}{c}\text { Number of } \\
\text { dormant nodes }\end{array}$ \\
\hline \hline 0 & 61 & 61 & 6 \\
\hline \hline 25 & 68 & 88 & 6 \\
\hline \hline 50 & 64 & 80 & 7 \\
\hline
\end{tabular}

To more illustrate results of above table, Fig (5) represent the coverage rate from network before and after optimization. The red line is represent the coverage rate in optimization while the blue line represent without optimization. The number of dormant nodes is respected to number of rounds of the two algorithms. The dormant nodes are put out the WSN coverage.

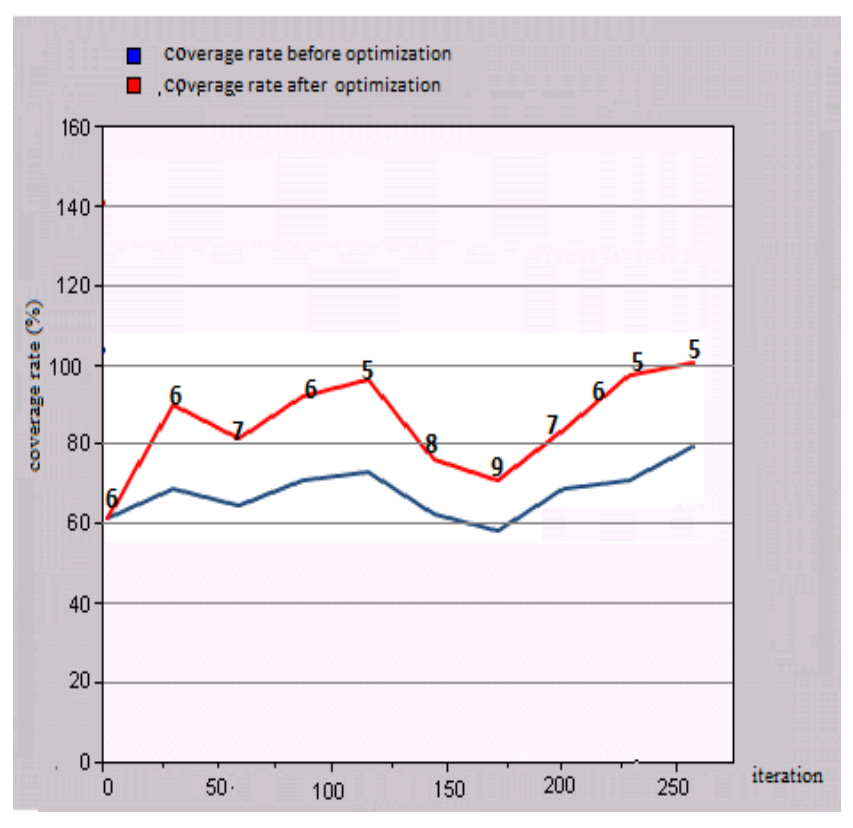

Fig (5): coverage rates before and after contrast optimization algorithm

Network reliability is become more stability in optimization algorithm also coverage rate is become more large in optimization method. The rate of change and the number of nodes covering dormant optimized, where in the initial coverage blue line before optimization random deployment of the network. A red line represents excellent after coverage of the figure indicates the number of times after the optimization dormant nodes.

\subsection{Experiments and Analysis}

This section is aimed to compare the tests obtained in this paper based on bat swarm mixed wireless network sensor(WSNBA) and the algorithm apply multi- objective optimization (WSNOP)[7]. For the same area to be monitored, each algorithm independently runs 10 times of iteration. The average numbers of iterations the evolutionary process and the corresponding coverage of the results shown in figure (5). 


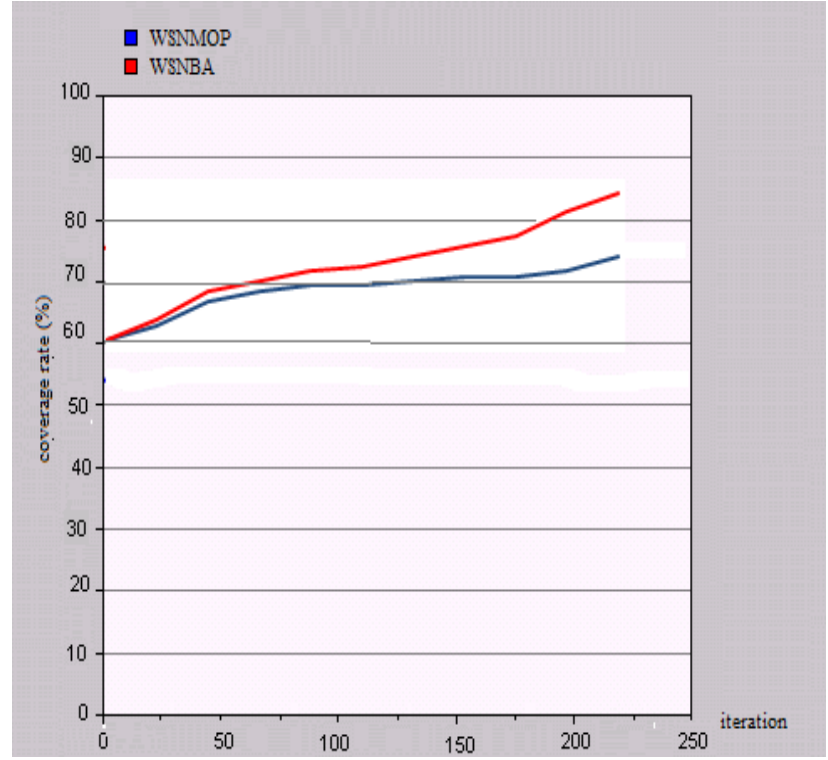

Fig (6): Coverage rate comparison

As seen in fig (6) shown coverage rate results and comparison for two algorithms. in early stages the converges faster slightly but after alteration coverage curve for WSNBA increased gradually for stabilize near certain. Also the WSNBA algorithm introduces multi-objective optimization of neighborhood disturbances. So iterative process lead to improve search results. Finally the proposed algorithm gets higher network coverage.

\subsection{Analysis of experimental results}

This paper is introduced multi-objective optimization for bat algorithm based on conflicts neighborhood for coverage area. It can enhance the performance of random coverage deployment for hybrid wireless sensor networks due to three reasons: First, algorithm BA is used for dynamic neighborhood conflicts through operation. The fitness will be strong perturbation amplitude small particles to enhance local search purposes, on other hand, increasing chances to find a better location. Secondly, algorithm based on elitist nondominated sorting strategy that provides a good Pareto sets. Finally, any bat is selected global optimal solution from current non-inferior solution by weighting centralized from Pareto optimal.

\section{CONCLUSION}

This paper is presented new approach for WSN coverage rate. The approach is achieved by using BA algorithm providing an effective multi-objective optimization to obtain reliable optimal rate coverage area. The proposed algorithm is designed to achieve non dominated Pareto optimization fronts which are uniformly spread and wide. WSNBA was based on neighborhood disturbances multi-objective bat algorithm can optimize for coverage performance of random deployment of hybrid wireless sensor networks. Solution of approach is compared to a public algorithm named WSNOP using an event-based simulator. Compression results show WSNBA algorithm is better performance from other. In the future: explore the technique to improve the convergence speed and analyze the parameters of the algorithm to reduce the effect of dormant nodes.

\section{REFERENCES}

[1] YUAN, Hao , LI Changbing, DU Maokang, 2012. Optimal Distribution of Nodes in Wireless Sensor
Network Based on Multi-objective Optimization” Journal of Computational Information Systems 8: 8 (3331-3338).

[2] X. F. Zou, Y. Chen, M. Z. Liu, et al., 2008. A New Evolutionary Algorithm for Solving Many-objective Optimization Problems [J]. IEEE Transaction on Systems, Man and Cybernetics, Part B, , 38 (5): 1402 1412.

[3] Liao, S.; Zhang, Q. A , 2013. Multi-Utility Framework with Application for Studying Tradeoff between Utility and Lifetime in Wireless Sensor Networks. IEEE Trans. Veh. Technol. , PP, doi: 10.1109 / TVT.. 2372793.

[4] Jameii Seyed Mahdi\& Jameii Seyed Mohsen 2013. Multi-objective Energy Efficient Optimization Agorithm for Coverage control in Wireless Sensor Networks. International Journal of Computer Science, Engineering and Information Technology (IJCSEIT), Vol.3,No.4.

[5] LYUDMILA M, DAVIDRB. 2011. Localization of Mobile Nodes in Wireless Networks with Correlated in Time Measurement Noise . IEEETRANS ACTIONSON MOBILE COMPUTING, ,10(1):44-53.

[6] Vinothini M, Umamakeswari, 2014. A Reliable data transmission using efficient neighbor coverage routing protocol in wireless sensor network. Indian $\mathbf{J}$ Sci Technol.; 7(12):2118-23.

[7] Singh Vinay Kumar \& Sharma Vidushi , 2013. A Multiobjective Coverage and Connectivity Strategy for Improving the Performance of Wireless Sensor Networks, International Journal of Advanced Research in Computer Science and Software Engineering Volume 3, Issue 12, ISSN: 2277 128X.

[8] Fidanova Stefka and Marinov Pencho. 2014. Multiobjective ACO algorithm for WSN layout: performance according to number of ants .Int. J. Metaheuristics, Vol. 3, No. 2 .

[9] R K Jena1 and P K Mahbati ,2012 Node Placement for Wireless Sensor Network Using Multi-objective PSO. International Conference on Computer Technology and Science) IPCSIT vol. 47 IACSIT Press, Singapore DOI: 10.7763/IPCSIT.

[10] Jena R K, 2014， Artificial Bee Colony Algorithm based Multi-Objective Node Placement for Wireless Sensor Network. I JTCS. Information Journal Technology and Computer Science, , 06, 25-32.

[11] Sengupta, S.; Das, S.; Nasir, M.; Panigrahi, B.K. Multiobjective node deployment in WSNs: In search of an optimal trade-off among coverage, lifetime, energy consumption, and connectivity. Eng. Appl. Artif. Intell., 26, 405-416.

[12] G. Molina, E. Alba, and E.-G. Talbi, 2008 Optimal sensor network lay out using multi-objective metaheuristics .J. Univer. Comput. Sci., vol. 14, no. 15, pp. 2549-2565.

[13] Gorain Barun, Mandal Partha Sarathi, 2014 Approximation algorithms for sweep coverage in wireless sensor networks. Journal of Parallel and Distributed Computing 74(8) 2699-2707,. 
[14] Yang Xin-She , Deb Suash , Fong Simon," Bat Algorithm is Better Than Intermittent Search Strategy" Journal of Multiple -Valued Logic and Soft Computing.

[15] Jiang Fei, 2014 . Application of hybrid ant colony algorithm in wireless sensor network coverage. computer modeling \& new technologies 18(12A) 161-166.

[16] Anand D.G., Chandrakanth H.G. \& Giriprasad M.N. 2011. Energy Efficient Coverage Problems in Wireless Ad Hoc Sensor Networks" Advanced Computing. An International Journal ( ACIJ ), Vol.2, No.2, March.

[17] Taha, Ahmed \& Tang Alicia Y.C. 2013. Bat Algorithm for Rough set Attribute Reduction. Journal of Theoretical and Applied Information Technology 10th May. Vol. 51 No.1 2005 - 2013. ISSN: 1992-8645.
[18] Jr Iztok Fister, Fister Du`san, Yang Xin-She , , 2013. A Hybrid Bat Algorithm, ELEKTROTEHNISKI VESTNIK 80(1-2): 1-7.

[19] Wang Gai-Ge \& Chang Bao2015. A Multi-Swarm Bat Algorithm for Global Optimization. IEEE press 978-14799-7492-4/15/\$31.00,

[20] Singh Vinay Kumar \& Sharma Vidushi, 2013 .A Multiobjective Coverage and Connectivity Strategy for Improving the Performance of Wireless Sensor Networks. International Journal of Advanced Research in Computer Science and Software Engineering Volume 3, Issue 12, DecemberISSN: 2277 128X.

[21] Al-Mukhtar Mumtaz M. Ali \& Hadi Teeb Hussein, 2014. Monitoring System Using Wireless Sensor Network. Journal of Al-Nahrain University Vol.17 (2), June, , pp.219-226. 